ency on each other. In biological terms this means that there exists no way by which a statistical analysis can open up the ring of VOL $\leftrightarrow$ GFR $\leftrightarrow R P F \leftrightarrow$ VOL and tell us which is cause and which are effects. The multiple coefficients of correlation in the rings are 0.85 and 0.79 in diabetics and controls, respectively (Fig. 1).

Focussing on GFR - the variable we want to explain - we may analyze the contribution from each of the variables VOL and RPF if we assume a direction of their interaction: Either variation in kidney size is the cause of variation in total kidney perfusion or vice versa but not both. ('Cause of' is now used in a strict statistical sense). Again, the statistical analysis in itself cannot give clues to the distinction between these alternatives. However, in a rational biological universe it hardly makes sense to consider perfusion as the cause of organ size.

Under this model, variation in kidney size explains $54 \%$ of the variation observed in GFR in diabetics and RPF explains an additional $19 \%$ whereas the remaining $27 \%$ is unexplained (Fig. 1 ).

It is worth noticing that in all studies the strength of the true relationship between GFR and RPF was overestimated due to the dependency of the two estimates on the same voided volume of urine. Moreover, the strength of the true relationship between kidney size and either of the two functional parameters was most likely underestimated since in two studies $[1,3]$ kidney volume was only calculated from the area of the $\mathrm{X}$-ray projected kidney and the remaining study the volume of one kidney was taken to be exactly half of the sum of that of two kidneys.

The biological conclusion to be drawn from the statistical analysis of the combined studies is therefore that the increased kidney size in diabetics is the major determinant for the increased GFR and it may even be the sole determinant. This is in complete accord with a recent study in short-term diabetics [6] in which the much more direct relationship between GFR and the absolute area of the glomerular filtration surface (GFS) was studied and where variations in GFS were shown to leave only $14 \%$ of the variation in GFR unexplained.
The above mentioned quite small fractions of unexplained variation in GFR should be interpreted only to mean that any other factor or variable governing GFR cannot do so independent of the kidney's structural quantities.

\section{H. J. G. Gundersen and C. E. Mogensen}

\section{References}

1. Mogensen CE, Andersen MJF (1973) Increased kidney size and glomerular filtration rate in early juvenile diabetes. Diabetes 22: 706-712

2. Sandahl Christiansen J, Gammelgaard J, Frandsen M, Parving H-H (1981) Increased kidney size, glomerular filtration rate and renal plasma flow in short-term insulin-dependent diabetics. Diabetologia 20: 451-456

3. Puig JG, Antón FM, Grande C, Pallardo LF, Arnalich F, Gil A, Vázquez JJ, García AM (1981) Relation of kidney size to kidney function in early juvenile diabetes. Diabetologia 21 : 363367

4. Diem K, Lentner C (1970) Documenta Geigy, Scientific tables, 7 th ed. Geigy, Basle, p 180

5. Nie $\mathrm{NH}$, Hull $\mathrm{CH}$, Jenkins JG, Steinbrenner K, Bent DH (1975) SPSS, 2nd edn. McGraw-Hill Book Company, New York, pp 334-340

6. Hirose K, Tsuchida H, Østerby R, Gundersen HJG (1980) A strong correlation between glomerular filtration rate and filtration surface in diabetic kidney hyperfunction. J Lab Invest 43: $434-437$

H. J. G. Gundersen

2nd University Clinic of Internal Medicine

Aarhus University

DK-8000 Aarhus C, Denmark

\title{
Plasma Non-Esterified Fatty Acid Levels and Atherogenesis in Diabetes Mellitus
}

Dear Sir,

Dr. Reinila's paper [1] relating elevated levels of plasma nonesterified fatty acids (NEFA) to morphological changes in the arteries of diabetic rats is of considerable interest. His data show that the severity of arterial lesions increased in parallel with the increase in the ratio of NEFA and albumin concentration in plasma, and that platelets tended to aggregate on the intima of arteries obtained from animals with the highest NEFA/albumin ratios. These observations are consistent with those of Gjesdal et al. [2], who demonstrated that in healthy volunteers fasted for prolonged periods, elevated NEFA concentrations $(1.8 \mathrm{mmol} / \mathrm{l})$ were associated with increased venous platelet aggregates and platelet factor 4 concentrations in the plasma. Our recent observations that albumin in plasma has a prostacyclin protecting effect, and that NEFA may accelerate the rate of decay of prostacyclin in plasma may provide one mechanism to explain the increased platelet aggregability and the vascular changes associated with increased NEFA/albumin ratios. The experimental procedure undertaken to elucidate this effect of fatty acids on prostacyclin decay is described below.

Prostacyclin ( $\mathrm{PGI}_{2}$; final concentration $20 \mathrm{ng} / \mathrm{ml}$ ) was added to a solution of human fatty acid free albumin $(40 \mathrm{~g} / 1)$ in phosphate buffer (final $\mathrm{pH} 7.4$ ) and incubated at $37^{\circ} \mathrm{C}$. Aliquots were taken at various time intervals and added to platelet rich plasma. After
Table 1. Effect of albumin and albumin + palmitic acid upon the decay of anti-aggregatory activity of prostacyclin $\left(\mathrm{PGI}_{2}\right)$ in a buffer solution

\begin{tabular}{lllll}
\hline $\begin{array}{l}\text { In- } \\
\begin{array}{l}\text { cubation } \\
\text { time } \\
\text { (min) }\end{array}\end{array}$ & $\begin{array}{l}\text { Buffer } \\
+\mathrm{PGI}_{2}\end{array}$ & $\begin{array}{l}\text { Fatty acid } \\
\text { free albumin } \\
+\mathrm{PGI}_{2}\end{array}$ & $\begin{array}{l}\text { Fatty acid } \\
\text { free albumin } \\
+\mathrm{PGI}_{2} \\
+1 \mathrm{mmol} / 1 \\
\text { palmitic acid }\end{array}$ & $\begin{array}{l}\text { Fatty acid } \\
\text { free albumin } \\
+\mathrm{PGI}_{2} \\
+2 \mathrm{mmol} / 1\end{array}$ \\
\hline 5 & 2 & 0 & 1 & 10 \\
10 & 39 & 3 & 6 & 25 \\
20 & 62 & 7 & 18 & 56 \\
30 & 75 & 13 & 40 & 68 \\
40 & 79 & 22 & 62 & 75 \\
60 & 79 & 51 & 77 & 78 \\
80 & 80 & 65 & 80 & 83 \\
100 & 77 & 75 & 77 & 76 \\
120 & 75 & 81 & 82 & 80 \\
\hline
\end{tabular}

Results are expressed as percentage aggregation after incubation with the substances mentioned above and the addition of ADP $(10 \mu \mathrm{mol} / \mathrm{l})$. The values expressed represent the mean of four observations, each obtained from one normal subject 
1 min incubation, aggregation was induced with adenosine diphosphate (ADP; final concentration $10 \mu \mathrm{mol} / \mathrm{l}$ ). Aggregation was recorded, at $37^{\circ} \mathrm{C}$, as the fall in optical density 3 min after adding ADP. The decay of $\mathrm{PGI}_{2}$ anti-aggregatory activity with time was compared with that of $\mathrm{PGI}_{2}$ incubated in phosphate buffer alone (also at $\mathrm{pH}$ 7.4). The rate of decay of $\mathrm{PGI}_{2}$ was markedly retarded in the presence of fatty acid free albumin (Table 1). The addition of palmitic acid (final concentrations 1 and $2 \mathrm{mmol} / \mathrm{l}$ ) to fatty acid free albumin and $\mathrm{PGI}_{2}$ solutions reduced this 'protective' effect markedly in a dose dependent fashion (Table 1). Similar results were obtained by adding oleic, stearic, linolenic and linoleic acids.

Our observations in vitro indicate clearly that NEFA and albumin in plasma have opposite effects on prostacyclin decay. Whether increasing NEFA concentrations or NEFA/albumin ratios exert a similar effect in vivo remains to be demonstrated.

Yours sincerely,

D. P. Mikhailidis, A. M. Mikhailidis, and P. Dandona

\section{References}

1. Reinila A (1981) Long-term effects of untreated diabetes on the arterial wall in rat. Diabetologia 20:205-212

2. Gjesdal K, Nordoy A, Wang H, Bernstein H, Mjøs OD (1976) Effects of fasting on plasma and platelet-free fatty acids and platelet function in healthy males. Thrombosis Haemostasis 36: $325-333$

\author{
P. Dandona \\ Metabolic Unit \\ Department of Chemical Pathology \\ Royal Free Hospital School of Medicine \\ Pond Street \\ London NW3 2QG, UK
}

\section{Book Reviews}

J. Irvine (Ed.): Immunology of Diabetes. Teviot Scientific Publications, Edinburgh, 1980 . Hardback: 377 pages. $£ 29.00$

This volume is based on a series of papers given at a symposium held in Edinburgh in 1978. Its delayed publication will not, fortunately, prevent it being a source book of considerable value for anyone involved in clinical research into diabetes. The major focus of interest is inevitably the possible role of immunological mechanisms in the pathogenesis of Type 1 diabetes and the evidence for this is authoritatively covered in several sections (unfortunately, there is no reference in the introductory survey to the contribution of Richard Lendrum, who first demonstrated the association between ICA and the onset of Type 1 diabetes and the importance of using Group 0 pancreatic tissue as substrate). The evidence for HLA-linked susceptibility genes is presented in several contributions, but the genetics of Type 1 diabetes is currently far from being completely understood and those particularly interested should also read more recently published analyses. There is coverage of the possible role of viruses in provoking the onset of Type 1 diabetes and a useful review of the various animal models. The evidence that immunological mechanisms may have a role in diabetic complication is presented. Considerable attention is given to the question of the classification of Type 1 diabetes but, as there is only a partial understanding of the way in which the various aetiological factors might interact in different patients, it would be wise to avoid dogmatism and to accept that any current classification can only be an interpretation of incomplete evidence and that it will require modification as further information becomes available. Anyone interested in diabetes research should find something of considerable interest in this book.
W.J. Malaisse and I.-B. Taljedal (Eds.): Biochemistry and Biophysics of the Pancreatic B Cell Hormone and Metabolic Research Supplement Series No. 10 European Workshop, Brussels 1979. Georg Thieme Verlag, Stuttgart, 1980. Paperback, 172 pages 124 illus., 51 tables, DM 80,00

On two occasions in the past (at Uppsala in 1964 and Umeå in 1969), meetings have been organized to bring together leading European workers in the field of pancreatic islet B cell metabolism and insulin secretion. The papers presented have subsequently been published, and in each case the resulting volume has served, and still serves, as a reference text for those needing to review the state of the work at that time. Since 1970 , spurred on by the ready availability of isolated islets for biochemical and biophysical studies, there has been an explosion of activity in this area and it is perhaps surprising that it was not until 1979 that a comparable meeting was again organised, this time as a result of the organisational and fund-raising skills of Dr. Willy Malaisse and his collaborators in Brussels. This book, which appears as a Supplement to Hormone and Metabolic Research, contains the texts of 27 papers presented at that meeting by leading European workers in this field, and as such provides a reasonable 'snapshot' of the state of research at that time. Readers, and in particular active research workers, may regret that the publishers have been unable to produce the volume until 18 months after the meeting so that the over-view, rather than the upto-date aspects of the resulting picture must be stressed. Nevertheless, like its predecessors, it should serve as an important reference work. These meetings have clearly fulfilled a useful purpose and it is to be hoped that the interval before the next one may be less than 10 years.

S. L. Howell (London)

\section{Erratum}

Diabetologia (1981) 21: 135-142 (No. 2, August)

Paper: Sener et al. "The Stimulus-Secretion Coupling of Amino Acid-Induced Insulin Release"

On page 140 , Table 6 , footnote ${ }^{\mathrm{b}}$, the units in parentheses should read $(132 \mathrm{nmol} / \mathrm{l})$ instead of $(132 \mathrm{mmol} / \mathrm{l})$. 